\title{
Aging Health: priorities in enhancing longevity
}

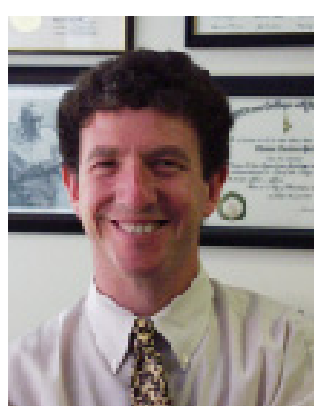

Thomas Perls ${ }^{\dagger}$

Boston University School of Medicine, Geriatrics Section, Department of Medicine, Boston, MA 02118, USA E-mail: thperls@bu.edu
'An academic journal dedicated to preventive and proactive strategies to help people age independently and to delay or escape age-related illness has been sorely needed. The birth of

Aging Health comes at a very opportune time.'

People vary a great deal in terms of how they age. Some seem to age relatively quickly and develop age-related illnesses such as heart disease or stroke in their forties and fifties, while others appear to age very slowly; and if they do develop age-related illnesses it may only be towards the end of their very long lives. Within these two extremes are the majority of people who, at least in industrialized nations, have an average life expectancy in their late seventies. Much of the variation around this average appears to be due to differences in environment, behavior and luck. As in the case of the Seventh Day Adventists, if an individual has good health habits, they are quite likely to live to an age that many humans appear to be capable of achieving - their late eighties (almost 30 years beyond the age of 60!) [1]. The HALE Study (Healthy aging: a Longitudinal study in Europe) assessed 10-year mortality amongst people aged 70-90 years in 11 European countries. Those who adhered to a Mediterranean diet, and moderate alcohol use, were physically active and refrained from smoking had a 50\% reduced mortality rate [2].

Unfortunately uncommon among people in industrial nations, the behavioral traits that facilitate survival to average life expectancy potential are regular exercise, a frugal vegetarian or Mediterranean diet, not being obese and not smoking. A number of studies suggest that effectively managing stress is also an important trait. Along these lines, Rebecca Levy, at Yale University, USA, has shown that being optimistic about one's aging also improves life expectancy [3]. When these behaviors are generally not adhered to, the result is an average life expectancy that is about 10 years less than what most of us should be capable of achieving. With that premature mortality, we likely also see more chronic illness and health-related costs to both individuals and to society. Thus, rather than aging being typified by the ageist 'the older you get, the sicker you get', the much more optimistic and enabling 'the older you get, the healthier you've been' is likely the case.

With disability-free years, older age can be some of the most valued and treasured years of a person's life. Thus, the first and likely realistic aim of longevity research should not be extending lifespan, but rather to extend what Jean Marie Robine terms active-life expectancy [4]. It appears the most potent way to achieve such a goal would be by emulating something akin to the Seventh Day Adventist life-style.

What then, does it take to live even longer, beyond the nonagenarian years, to the age of 100 years and older? First, it appears a resistance to disability is necessary. One study retrospectively found that nearly $90 \%$ of centenarians were independently functioning at an average age of 92 years [5]. On the other hand, many of these very old individuals lived with age-related illness(es) for a long period of time (unlike many other older people, who die of those diseases)[6]. Thus, gerontologists have become very interested in quantifying functional reserve and adaptive capacity, the means by which people can successfully contend with age-related illnesses; both quantifying these as well as deciphering their biological underpinnings are very important priorities for the development of therapeutic drugs and strategies.

Second, lacking environmental, behavioral, stochastic (bad luck), and genetic factors ('bad genes') that predispose to poor health would be important to achieve exceptional survival. Genetic findings related to human longevity have, thus far, primarily been related to vascular health $[7,8]$. This is not surprising given that vascular disease is the number one killer among older people, including centenarians. One would expect that the oldest old would have to relatively lack genetic variations that predispose to vascular disease at an earlier age.

More elusive has been the search for human genetic variations that impact upon the basic biology of aging and which could impact upon 
the rate of aging and susceptibility to a broad range of age-related diseases. Discovering such so-called 'longevity-enabling' genes might entail the study of the extreme of old age such as supercentenarians, those who live to 110 years old and older or those who achieve extreme old age despite habits that would otherwise be associated with high mortality and age-associated disease risk, such as smoking. In the case of lower organisms, scientists have focused on genes that participate in insulin signalling pathways and the human homologs of these genes may be important candidates for study among humans. Delineating the genes and pathways that play important roles in the basic biology of aging could also lead to the discovery of therapeutics that slow aging and decrease susceptibility to age-related diseases. However, the development of such drugs is likely to be many years away and potentially accessible to relatively few people. Thus, long-term changes in behaviors that impact upon longevity is by far and away going to provide our biggest bang for the buck in terms of dedicating resources to improve public health.

An academic journal dedicated to preventive and proactive strategies to help people age independently and to delay or escape age-related illness has been sorely needed. The birth of Aging Health comes at a very opportune time. As you can see by the articles appearing in this inaugural issue of Aging Health, numerous strategies from potential stem-cell-based therapies to vaccines are becoming real possibilities. As clinicians and scientists turn more and more of their attention to illnesses of old age, we are also seeing how these diseases are diagnosed and approached using strategies different from those used with middle-aged adults. These strategies are also prominently discussed in Aging Health. The multi-disciplinary approach that is so important to good geriatric care makes the commingling of articles spanning the basic and clinical sciences certainly appropriate and effective for an audience interested in aging health.

\section{Bibliography}

1. Fraser GE, Shavlik DJ: Ten years of life: Is it a matter of choice? Arch. Intern. Med. 161(13), 1645-1652 (2001).

2. Knoops KTB, de Groot LC, Kromhout D et al:: Mediterranean Diet, Lifestyle Factors, and 10-Year Mortality in Elderly European Men and Women. The HALE Project. JAMA 292, 1433-1439 (2004).

3. Levy BR, Slade MD, Kunkel SR, Kasl SV: Longevity increased by positive self- perceptions of aging. J. Personality Social Psychol. 83, 261-270 (2002).

4. Robine JM, Michel JP: Looking forward to a general theory on population aging. $J$. Gerontol. A Biol. Sci. Med. Sci. 59, M590M597 (2004).

5. Hitt R, Young-Xu Y, Silver M, Perls T: Centenarians: the older you get, the healthier you have been. Lancet 354(9179), 652 (1999).

6. Evert J, Lawler E, Bogan H, Perls T: Morbidity profiles of centenarians: survivors, delayers, and escapers. J. Gerontol. A Biol. Sci. Med. Sci. 58(3), 232-237 (2003).

7. Barzilai N, Schechter C, Schaefer EJ et al: Unique lipoprotien phenotype and genotype in humans with exceptional longevity. JAMA 290(15), 2030-2040 (2003).

8. Schachter F, Faure-Delanef L, Guenot F et al:: Genetic associations with human longevity at the APOE and ACE loci. Nature Genet. 6(1), 29-32 (1994). 